\title{
Short-sequence superadhesive peptides with topologically enhanced cation- $\pi$ interactions
}

Heng Chang a,bł, Vahid Adibnia ${ }^{b \ddagger}$, Chuanxi Li ${ }^{\text {a }}$, Rongxin Su ${ }^{\text {a,c,d*}}$, Wei Qi ${ }^{\text {a,c }}$, and Xavier Banquy ${ }^{b *}$

a State Key Laboratory of Chemical Engineering, Tianjin Key Laboratory of Membrane Science and Desalination Technology, School of Chemical Engineering and Technology, Tianjin University, Tianjin 300072, China.

b Faculty of Pharmacy, Université de Montréal, 2900 Edouard-Montpetit, Montréal, Québec H3C 3J7, Canada.

${ }^{c}$ Collaborative Innovation Center of Chemical Science and Engineering (Tianjin), Tianjin 300072, China.

d School of Marine Science and Technology, Tianjin University, Tianjin 300072, China. 


\section{Supplementary figures}
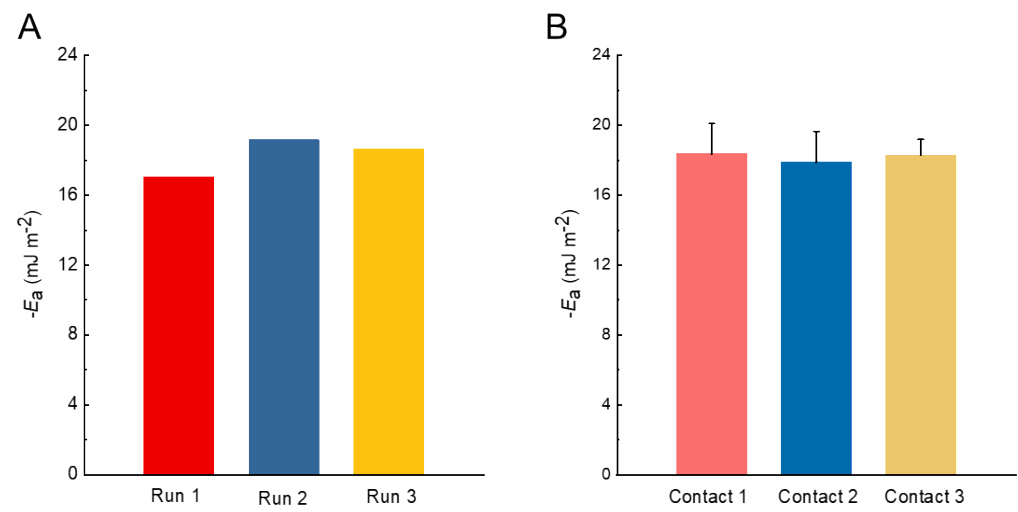

Figure S1. (A) The adhesive energy of PEP 1 from different force runs measured on one contact position. (B) The adhesion energy of PEP 1 measured at different contact positions multiple timesm.
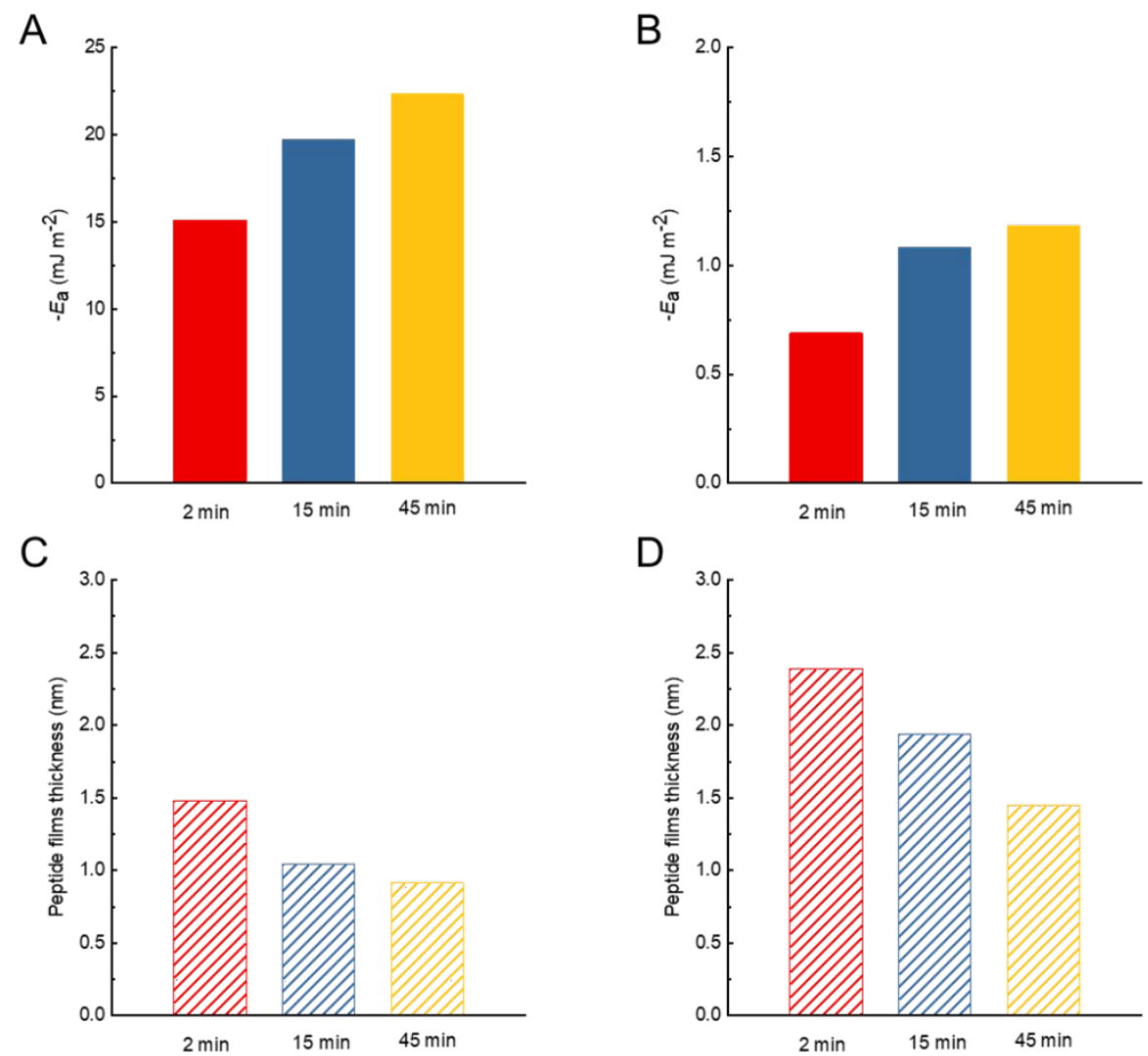

Figure S2. The effects of contact time on the adhesive energy of the PEP 1 (A) and PEP 2 (B) films. The effects of contact time on the thickness of peptide films of PEP 1 (C) and PEP 2 (D). 
A

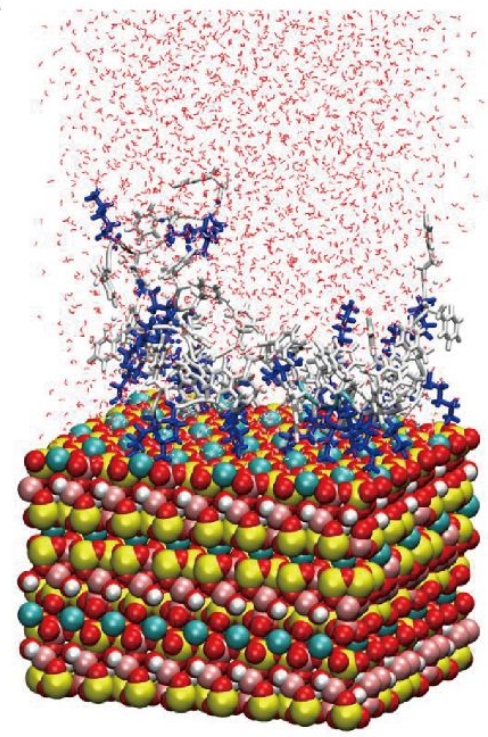

B

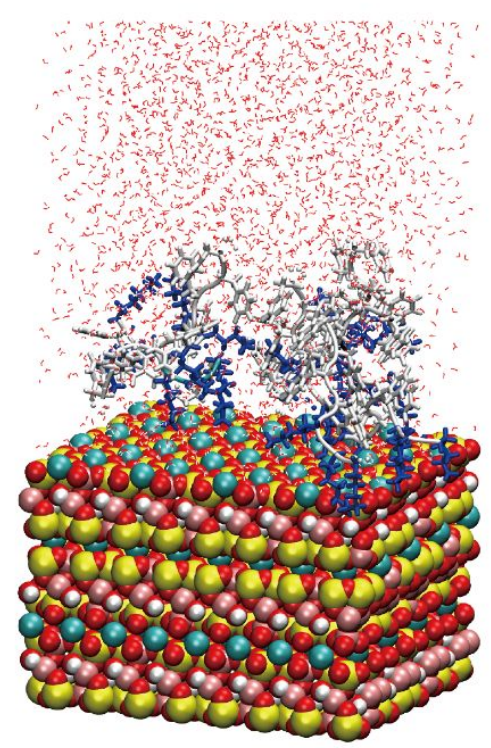

Figure S3. Representation of PEP 1 (left) and PEP 3 (right) adsorbed on mica underwater from MD simulations. Blue shows lysine amino acids and white shows phenylalanine amino acids. Water molecules are shown in red.

A

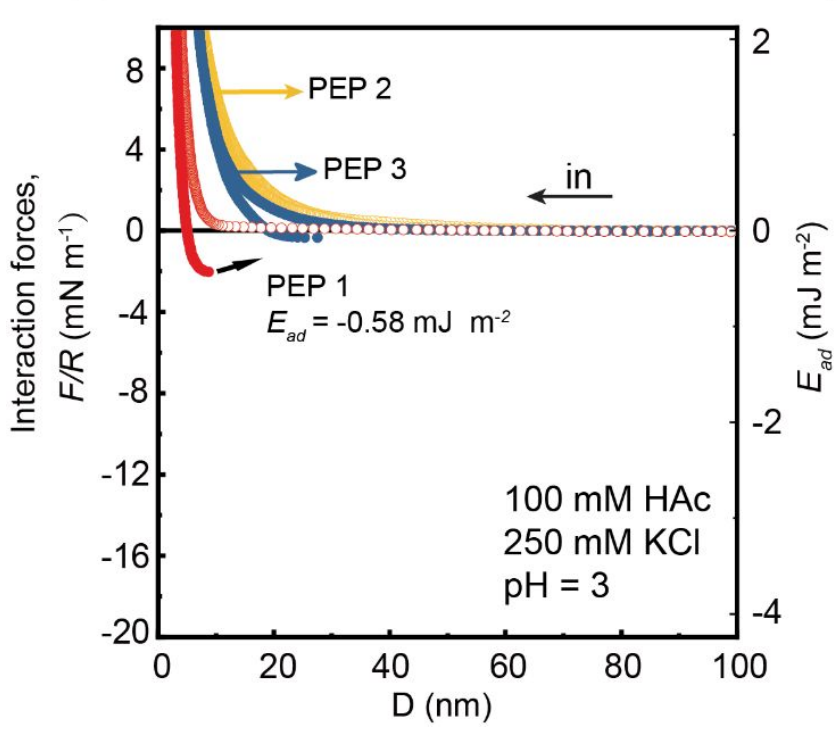

B

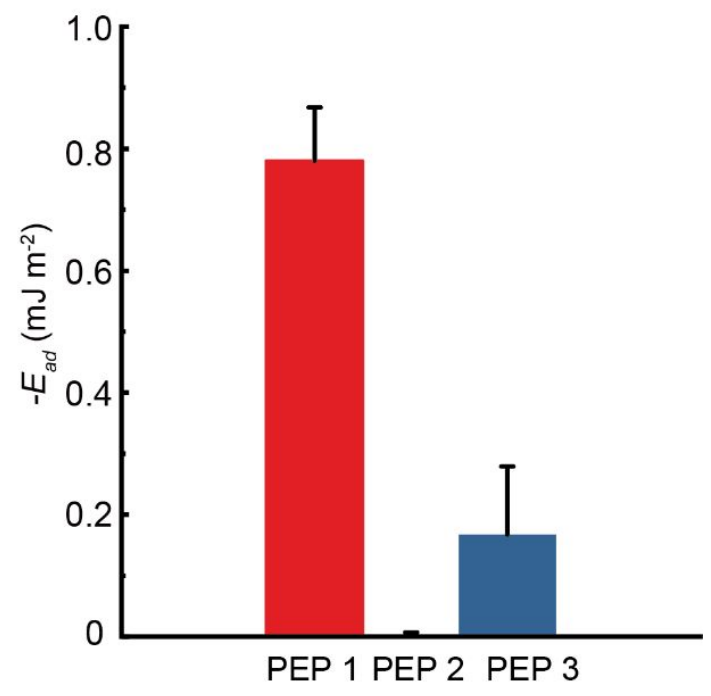

Figure S4. (A) Force-distance profiles for peptide films in a $100 \mathrm{mM}$ acetic acid solution with $250 \mathrm{mM}$ $\mathrm{KCl}$. (B) Adhesion energy obtained from the force profiles for each peptide in acid and salt media. 


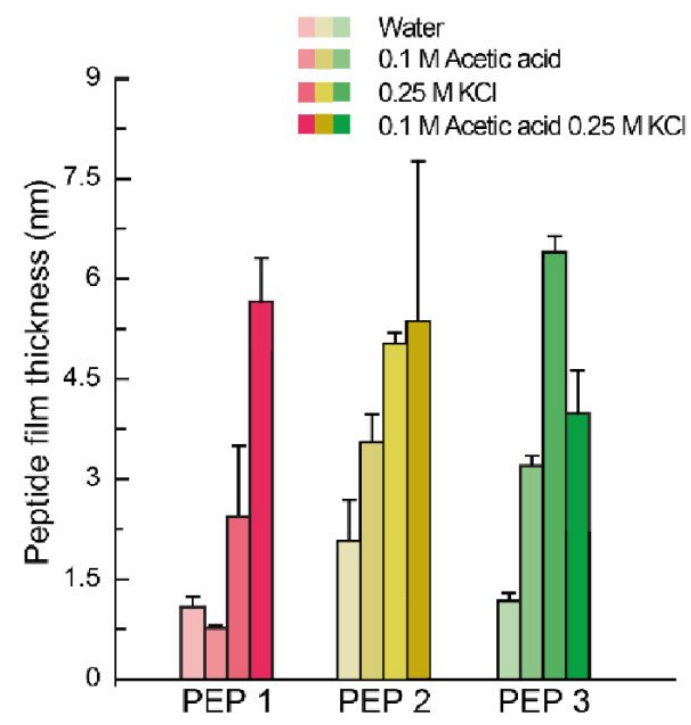

Figure S5. Hardwall thickness of the peptide films in different media.
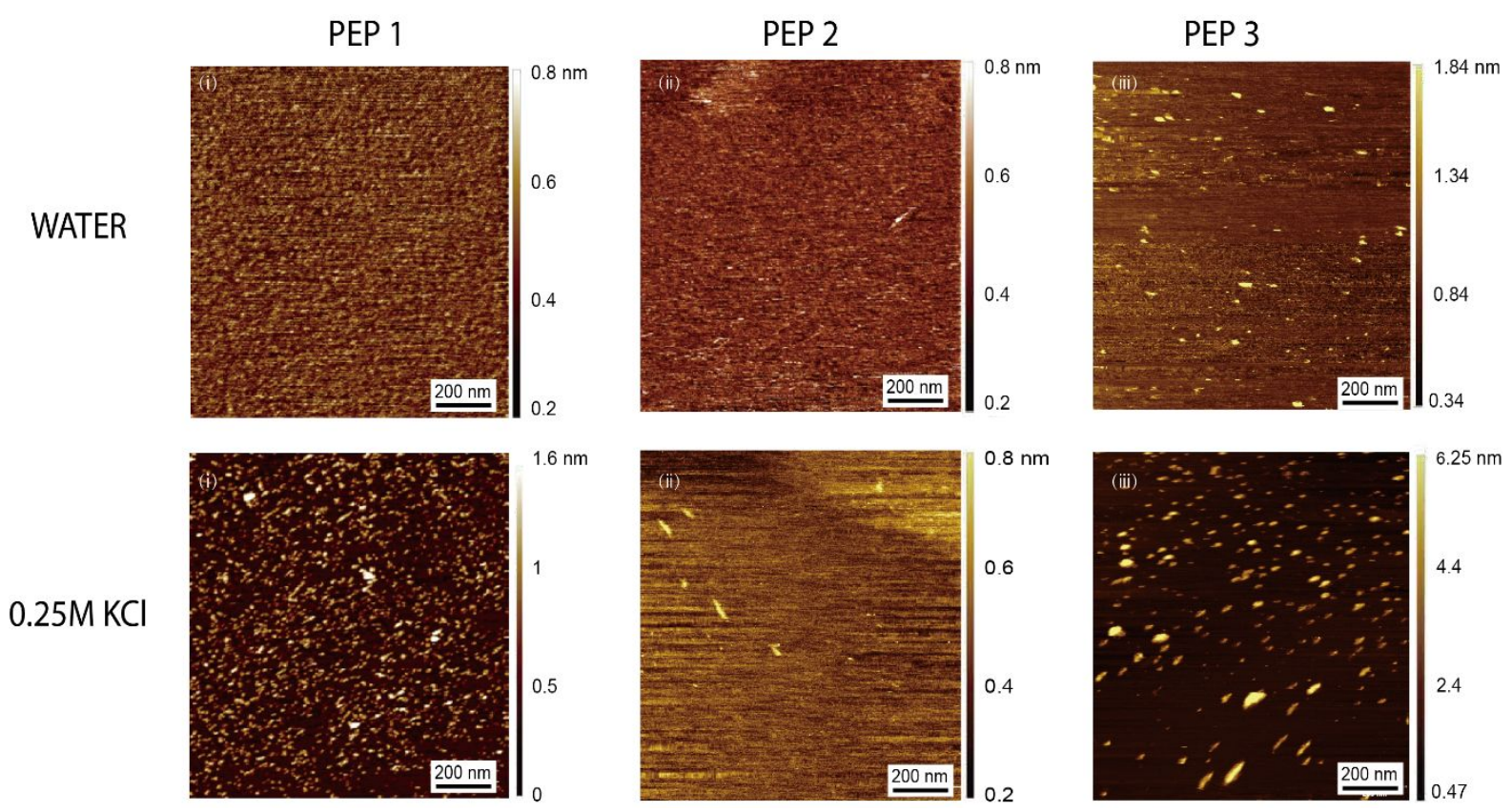

Figure S6. The AFM images of PEP-1, PEP-2 and PEP-3 in water (top) and salt (bottom) solutions. 
Table S1. Adhesion energy of underwater adhesives used in Figure 5.

\begin{tabular}{|c|c|c|c|c|}
\hline Reference & System & $\begin{array}{c}\text { Adhesion } \\
\text { mechanism }\end{array}$ & $\begin{array}{c}E_{\mathrm{ad}}\left(\mathrm{mJ} \mathrm{m^{- }}\right. \\
2)\end{array}$ & Test Environment \\
\hline & $\begin{array}{l}\text { recombinant Mytilus } \\
\text { foot protein- } 1\end{array}$ & cation $-\pi$ & $\sim 4$ & $\begin{array}{c}0.1 \mathrm{mM} \mathrm{KNO}_{3}, 0.1 \mathrm{M} \\
\text { acetic acid, pH } 3.0\end{array}$ \\
\hline $\begin{array}{l}\text { Kim et al. } \\
(2015)^{1}\end{array}$ & $\begin{array}{l}\text { decapeptide of } \\
\text { recombinant Mytilus } \\
\text { foot protein- } 1\end{array}$ & cation $-\pi$ & $\sim 6$ & $\begin{array}{c}0.1 \mathrm{mM} \mathrm{KNO}_{3}, 0.1 \mathrm{M} \\
\text { acetic acid, pH } 3.0\end{array}$ \\
\hline $\begin{array}{l}\text { Kim et al. } \\
(2017)^{2}\end{array}$ & $\begin{array}{l}\text { recombinant Mytilus } \\
\text { foot protein- } 1\end{array}$ & cation $-\pi$ & $\sim 3.3$ & $0.1 \mathrm{M}$ acetic acid, pH 3.0 \\
\hline \multirow{3}{*}{$\begin{array}{l}\text { Lu et al. } \\
(2013)^{3}\end{array}$} & $\begin{array}{l}\text { poly-L-lysine and poly- } \\
\text { L-tryptophan }\end{array}$ & cation $-\pi$ & 1.9 & 0.05M acetic acid, $\mathrm{pH} 3.0$ \\
\hline & $\begin{array}{l}\text { poly-L-lysine and } \\
\text { polystyrene }\end{array}$ & cation $-\pi$ & 1.3 & 0.05M acetic acid, $\mathrm{pH} 3.0$ \\
\hline & $\begin{array}{l}\text { poly-L-lysine and poly- } \\
\text { L-tyrosine }\end{array}$ & cation $-\pi$ & 0.7 & 0.05M acetic acid, $\mathrm{pH} 3.0$ \\
\hline $\begin{array}{l}\text { Lim et al. } \\
(2016)^{4}\end{array}$ & poly(norepinephrine) & $\begin{array}{l}\pi-\pi \text { and cation }-\pi \\
\text { interactions }\end{array}$ & 1.35 & 10 mM PBS buffer, $\mathrm{pH} 8.4$ \\
\hline $\begin{array}{l}\text { Xie et al. } \\
(2019)^{5}\end{array}$ & Poly (MPC-co-DMA) & $\begin{array}{c}\text { cation }-\pi \text { and } \\
\text { hydrogen bonding } \\
\text { interactions }\end{array}$ & 4.3 & Acetic acid pH 4 \\
\hline \multirow{3}{*}{$\begin{array}{l}\text { Deepankum } \\
\text { ar et al. } \\
(2019)^{6}\end{array}$} & suckerin-12 & $\begin{array}{c}\text { unraveling of } \\
\text { intermolecular } \beta \text { - } \\
\text { sheets domains }\end{array}$ & $\sim 4$ & $0.1 \mathrm{M}$ acetic acid, $\mathrm{pH} 3.0$ \\
\hline & suckerin-12-Dopa & $\begin{array}{c}\text { unraveling of } \\
\text { intermolecular } \beta \text { - } \\
\text { sheets domains }\end{array}$ & $\sim 5.5$ & $0.1 \mathrm{M}$ acetic acid, pH 3.0 \\
\hline & Z-Cat-C10 & $\begin{array}{l}\text { hydrophobic } \\
\text { attraction and } \mathrm{H} \\
\text { bonding }\end{array}$ & 10.1 & DI water \\
\hline \multirow[t]{3}{*}{$\begin{array}{l}\text { Ahn et al. } \\
(2015)^{7}\end{array}$} & Z-Cat-C6 & $\begin{array}{l}\text { hydrophobic } \\
\text { attraction and } \mathrm{H} \\
\text { bonding }\end{array}$ & 9.6 & DI water \\
\hline & Z-Cat-Cat & $\begin{array}{l}\text { hydrophobic } \\
\text { attraction and } \mathrm{H} \\
\text { bonding }\end{array}$ & 8.1 & DI water \\
\hline & Z-Cat-C8 & $\begin{array}{l}\text { hydrophobic } \\
\text { attraction and } \mathrm{H} \\
\text { bonding }\end{array}$ & 2.5 & DI water \\
\hline
\end{tabular}




\section{Reference}

1. Kim, S.; Faghihnejad, A.; Lee, Y.; Jho, Y.; Zeng, H.; Hwang, D. S. Cation- $\pi$ Interaction in DOPA-Deficient Mussel Adhesive Protein Mfp-1.J. Mater. Chem. B 2015, 3, 738-743.

2. Kim, S.; Yoo, H. Y.; Huang, J.; Lee, Y.; Park, S.; Park, Y.; Jin, S.; Jung, Y. M.; Zeng, H.; Hwang, D. S. Salt Triggers the Simple Coacervation of An Underwater Adhesive When Cations Meet Aromatic $\pi$ Electrons in Seawater. ACS Nano 2017, 11, 6764-6772.

3. Lu, Q.; Oh, D. X.; Lee, Y.; Jho, Y.; Hwang, D. S.; Zeng, H., Nanomechanics of Cation- $\pi$ Interactions in Aqueous Solution. Angew. Chem.Int. Ed. 2013, 125, 4036-4040.

4. Lim, C.; Huang, J.; Kim, S.; Lee, H.; Zeng, H.; Hwang, D. S. Nanomechanics of Poly (Catecholamine) Coatings in Aqueous Solutions. Angew. Chem.Int. Ed. 2016, 55, 3342-3346.

5. Xie, L.; Gong, L.; Zhang, J.; Han, L.; Xiang, L.; Chen, J.; Liu, J.; Yan, B.; Zeng, H. A Wet Adhesion Strategy via Synergistic Cation- $\pi$ and Hydrogen Bonding Interactions of Antifouling Zwitterions and MusselInspired Binding Moieties. J. Mater. Chem. A 2019, 7, 21944-21952.

6. Deepankumar, K.; Lim, C.; Polte, I.; Zappone, B.; Labate, C.; De Santo, M. P.; Mohanram, H.; Palaniappan, A.; Hwang, D. S.; Miserez, A., Supramolecular $\beta$-Sheet Suckerin-Based Underwater Adhesives. Adv. Funct. Mater. 2020, 30, 1907534.

7. Ahn, B. K.; Das, S.; Linstadt, R.; Kaufman, Y.; Martinez-Rodriguez, N. R.; Mirshafian, R.; Kesselman, E.; Talmon, Y.; Lipshutz, B. H.; Israelachvili, J. N. High-Performance Mussel-Inspired Adhesives of Reduced Complexity. Nat. Commun. 2015, 6, 1-7. 